\title{
Varicella Zoster Virus Vasculopathy
}

\author{
Maria A. Nagel ${ }^{1,2}$ and Andrew N. Bubak ${ }^{1}$ \\ Departments of ${ }^{1}$ Neurology and ${ }^{2}$ Ophthalmology, University of Colorado School of Medicine, Aurora
}

Varicella zoster virus (VZV) is a ubiquitous, exclusively human alphaherpesvirus that produces varicella then becomes latent in ganglionic neurons. In elderly and immunocompromised individuals, VZV reactivates and typically produces herpes zoster. Studies of patients with VZV vasculopathy have identified key clinical, imaging, and laboratory features to assist in diagnosis and treatment. Complementary studies have further expanded the spectrum of VZV vasculopathy to include the extracranial circulation and identified mechanisms contributing to its pathogenesis. Given our increasing aging population and recognition that VZV reactivation manifesting as zoster is a risk factor for stroke and myocardial infarction, recognition of VZV as a potential cause of vascular disease with or without associated zoster rash is essential to decrease associated morbidity and mortality because VZV vasculopathy can be treated with antiviral therapy.

Keywords. VZV; vasculopathy; vasculitis.

Varicella zoster virus (VZV) is an exclusively human neurotropic alphaherpesvirus that infects more than $95 \%$ of the US population. Primary infection produces varicella, followed by establishment of virus latency in cranial nerve, dorsal root, and autonomic ganglionic neurons [1]. With a decline in VZVspecific cell-mediated immunity in elderly and immunocompromised individuals, virus reactivates and produces herpes zoster, which can be complicated by postherpetic neuralgia. The burden of disease is significant given that $50 \%$ of individuals reactivate and develop zoster by 85 years of age.

VZV can also reactivate from trigeminal and autonomic ganglia of the head and neck and travel transaxonally to cerebral arteries where nerves terminate in the adventitia. Following virus deposition, persistent inflammation may occur, resulting in pathological vascular remodeling and stroke. VZV vasculopathy was first described in 1896 [2] and included cases of varicella or zoster that were temporally associated with stroke, particularly when zoster occurred in the ophthalmic division of the trigeminal nerve. Subsequently, affected cerebral arteries from patients with VZV vasculopathy were examined postmortem and found to have VZV DNA, VZV antigen, and herpesvirus particles [3], thus demonstrating that VZV vasculopathy was due to productive virus infection of arteries.

Finally, several epidemiological studies have shown that VZV reactivation, manifesting as zoster, is a risk factor for stroke particularly in individuals who develop zoster under 40 years of age, consistent with central spread of VZV to arteries in addition to peripheral spread to skin (reviewed in [4]). Furthermore,

Correspondence: M. A. Nagel, MD, Department of Neurology, University of Colorado School of Medicine, 12700 E. 19th Avenue, Mail Stop B182, Aurora, C0 80045 (maria.nagel@ucdenver.edu). The Journal of Infectious Diseases ${ }^{\circledR} \quad$ 2018;218(S2):S107-12

(C) The Author(s) 2018. Published by Oxford University Press for the Infectious Diseases Society of America. All rights reserved. For permissions, e-mail: journals.permissions@oup.com. DOI: 10.1093/infdis/jiy425 antiviral therapy during zoster appears to decrease this stroke risk [5], whereas zoster vaccination did not [6].

\section{CLINICAL FEATURES, LABORATORY ABNORMALITIES, DIAGNOSIS, ANDTREATMENT OF VZV VASCULOPATHY}

Historically, VZV vasculopathy was characterized as involving intracranial arteries and presented as transient ischemic attacks and ischemic or hemorrhagic strokes. In 30 patients with virologically confirmed VZV vasculopathy (ie, presence of VZV DNA or intrathecal synthesis of anti-VZV antibodies) [7], rash was present in $63 \%$, cerebrospinal fluid (CSF) pleocytosis was detected in $67 \%$, and the average time from rash to neurological symptoms and signs was 4.1 months. Brain imaging abnormalities were present in $97 \%$, typically seen as enhancing lesions at grey-white matter junctions. Of 23 patients analyzed by angiography, $70 \%$ had abnormalities predominantly in both large and small arteries (50\%), small arteries exclusively (37\%), and large arteries exclusively (13\%). Due to the protracted nature of disease, VZV DNA was detected in only $30 \%$ of CSF samples whereas anti-VZV IgG antibody was found in $93 \%$ of CSF samples, including a reduced serum/CSF ratio of anti-VZV IgG that confirmed intrathecal synthesis of anti-VZV IgG. While both polymerase chain reaction (PCR) for detection of VZV DNA and detection of antibody to VZV in CSF are highly specific, detection of anti-VZV IgG antibody in CSF is the more reliable test to diagnose VZV vasculopathy [7]. Overall, a positive PCR for VZV DNA in CSF can be diagnostic, but a negative PCR does not exclude the diagnosis; only negative results in both VZV PCR and anti-VZV IgG antibody testing in CSF excludes the diagnosis of VZV vasculopathy. Finally, two-thirds of the VZV vasculopathy patients stabilized or improved with antiviral therapy. Often, the diagnosis of VZV vasculopathy is missed, and hence antiviral treatment is not administered, due to the lengthy time between the occurrence of rash and stroke, the 
absence of rash, or the absence of a pleocytosis and VZV DNA in CSF.

In children, postvaricella stroke is usually monophasic, typically presenting as an acute hemiparesis at, on average, 4 months after varicella [8]. Recently, the live attenuated varicella vaccine strain was shown to cause VZV vasculopathy in an immunodeficient child [9], confirming the need for caution in vaccinating potentially immunocompromised children. Less commonly, VZV vasculopathy can present as aneurysms with subarachnoid hemorrhage and sinus thrombosis [10,11].

VZV vasculopathy should be suspected in individuals, particularly if immunocompromised, who have had a stroke or aneurysm with: (1) a recent history of varicella or zoster, (2) recurrence of unclear cause with or without rash, or (3) unclear etiology and absence of stroke risk factors. The best test for diagnosis in these suspected cases is a lumbar puncture and examination of CSF for the presence of anti-VZV antibodies and VZV DNA as noted above. Our enzyme-linked immunosorbent assay (ELISA)-based laboratory test for the presence of antiVZV IgG and IgM antibodies on paired CSF and serum samples is conducted by the Measles, Mumps, Rubella and Herpesvirus Laboratory Branch/ National Center for Immunization and Respiratory Disease at the Centers for Disease Control and Prevention, Atlanta, Georgia. Patients with VZV vasculopathy are treated with $10-15 \mathrm{mg} / \mathrm{kg}$ intravenous acyclovir for 14 days based upon Level 2 class of evidence (systemic review of cohort studies or individual cohort studies) extrapolated from treatment studies of herpes simplex virus central nervous system disease and VZV with visceral involvement (reviewed in [12]), as well as expert opinions and case series without controls of VZV vasculopathy [7]. For recurrent disease, a second course may be required, particularly in immunocompromised patients, followed by oral antivirals for several months. Because histological specimens often demonstrate arterial inflammation and based upon our clinical experience, we concurrently administer prednisone, $1 \mathrm{mg} / \mathrm{kg}$ from days $1-5$ of the 14-day acyclovir course without the need for a subsequent steroid taper.

\section{GIANT CELL ARTERITIS}

Recently, several studies emerged indicating that VZV vasculopathy can also affect the extracranial circulation, including temporal arteries (TAs) from patients with giant cell arteritis (GCA). GCA is the most common systemic vasculitis in the elderly and is characterized by a constellation of symptoms including severe headache, scalp tenderness, and vision loss, as well as a history of jaw claudication, polymyalgia rheumatica, fever, night sweats, weight loss, fatigue, and elevated inflammatory markers (erythrocyte sedimentation rate [ESR] and C-reactive protein [CRP]). Diagnosis of biopsy-positive GCA (GCA-positive) is made by the presence of transmural inflammation, medial smooth muscle cell damage, and multinucleated giant/epithelioid cells in noncontiguous skip lesions of the temporal artery (TA biopsies). In many clinically suspect cases, TA biopsy may be pathologically negative (GCA-negative) and decision to treat is based upon clinical presentation. Because vision loss frequently occurs, patients are immediately treated with corticosteroids, with $50 \%$ relapsing after discontinuation of therapy or progressing to stroke and vision loss despite therapy. The cause of GCA was not clear but activation of vascular dendritic cells in the artery wall by an unknown antigen in the adventitia was proposed as an early step in disease progression [13].

Analyses to test for the possible role of VZV infection in GCA were motivated by the virtually identical pathological changes seen in patients with intracerebral VZV vasculopathy and GCA, as well as multiple case reports and series demonstrating an overlap between features of GCA and VZV vasculopathy [14-16]. Furthermore, TA biopsies of patients with herpes zoster ophthalmicus with delayed arteritis also revealed GCA pathology [17]. Multiple immunohistochemical studies were subsequently conducted to determine the frequency of VZV antigen in formalin-fixed, paraffin-embedded GCA-negative, GCA-positive, and normal TAs [18, 19]. A cumulative study [20] detected VZV antigen in 73/104 (70\%) GCA-positive TAs and in 58/100 (58\%) GCA-negative TAs; the presence of VZV antigen in these 2 groups was statistically significant $(P<.0001)$ compared to $11 / 61$ (18\%) normal TAs that contained VZV antigen. VZV DNA was detected by PCR in many VZV antigen-positive sections, as well as herpesvirus particles by electron microscopy [19]. Adventitial inflammation was seen adjacent to viral antigen in 26 (52\%) of 58 GCA-negative subjects whose TAs contained VZV antigen and no inflammation was seen in normal TAs containing VZV antigen, most likely reflecting subclinical reactivation in some people over age 50 or early disease, which may be influenced by host and other exogenous factors. Overall, the greater frequency of VZV in the adventitia than in media and intima in all groups (86\% of GCA-positive subjects and $95 \%$ of GCA-negative subjects) most likely reflects transaxonal transport of virus along afferent nerve fibers that innervate the TA after reactivation from ganglia.

Recent studies failed to detect VZV in GCA-positive TAs at the frequency of the aforementioned studies. These discrepancies can be explained largely through methodological differences-specifically, the lower number of TA slides examined. Gilden and colleagues $[19,20]$ prepared 100 consecutive slides from each patient's TA biopsy and immunostained every other one for VZV antigen (50 slides); 2 blinded readers identified slides that contained VZV antigen, and a TA was considered VZV antigen-positive if at least 1 of the 50 slides was considered VZV antigen-positive by both readers. VZV antigen-positive slides were scraped, DNA extracted, and PCR attempted to amplify low-quality VZV DNA from formalin-fixed tissue. Specifically, over a 3-year period with 3 full-time research assistants dedicated to acquisition of data, a total of 5200 slides were 
immunostained from 104 GCA-positive TAs and at least 1 slide from 73 unique TA biopsies contained VZV antigen, of which $40 \%$ contained VZV DNA; 5000 slides were immunostained from 100 GCA-negative TAs and at least 58 TA-unique slides contained VZV antigen, of which 18\% contained VZV DNA; and 3050 slides were immunostained from 61 normal TAs and at least 11 TA-unique slides contained VZV antigen, of which 33\% contained VZV DNA. Thus, it is not surprising that Muratore and colleagues [21] did not detect VZV antigen in GCA-positive TAs when they stained 1 slide compared to 50 slides/TA of 34 GCA-positive TA biopsies and then an additional 30 slides/ TA in a subset of these GCA-positive TA biopsies $(n=10)$; this group immunostained a total of 334 slides compared to 5200 in the Gilden study. VZV DNA was also not detected. Similarly, Procop and colleagues failed to find VZV DNA in 5 snap-frozen GCA-positive TA biopsies [22], which is most likely due to the low abundance of viral antigen in skip lesions. A more extensive study reported VZV antigen in 12\% of GCA-positive TA biopsies rather than 70\%; the researchers stained between 10 and 40 sections/TA at varying sites in 25 patients (250-1000 total slides) [23]; VZV DNA was not measured in this study. While some of these studies presented power calculations to justify their sample sizes, deviations from original methodologies can render them ineffective and actually result in lower power than the original study, especially when fewer slides per TA biopsy were stained. It is important to stress that the prevalence of detecting VZV antigen/DNA remains positively correlated with the number of sections examined, as detailed in [19]. Due to the nature of VZV being present in skip areas, it is imperative that immunohistochemical studies be conducted in numerous sections at varying sites per TA biopsy and that comparisons between studies investigating VZV prevalence be methodologically similar with respect to number/location of sections and immunohistochemical protocols.

Taking together the significant association of VZV antigen in TAs of patients with GCA-negative and GCA-positive TAs compared to normal TAs and the same pathologies and vascular presentations of GCA and VZV vasculopathy, VZV appears to trigger the immunopathology of a significant subset of GCA. In virologically verified cases that contain $\mathrm{VZV}$ antigen and/or VZV DNA in TA biopsies and/or have a recent history of zoster, antiviral therapy should be considered because $50 \%$ of GCA in general progress or relapse during corticosteroid therapy or taper, and oral antiviral therapy (acyclovir, valacyclovir, famciclovir) is low risk with the exception of nephrotoxicity; hence the risks and benefits of antiviral treatment in GCA patients with renal dysfunction need to be considered.

While there have been single-case reports of GCA patients whose TAs contain VZV responding favorably to antiviral therapy, larger prospective studies still need to be undertaken to determine the effectiveness of antiviral therapy with or without corticosteroids in cases of GCA associated with VZV infection; however, it is established that intracerebral cases of VZV vasculopathy improve or stabilize with antiviral therapy [7]. We currently treat GCA associated with VZV infection with prednisone, $1 \mathrm{mg} / \mathrm{kg}$, along with valacyclovir $1 \mathrm{~g}, 3$ times daily (level 5 class of evidence: expert opinion without explicitly critical appraisal, or based on physiology, bench research, or "first principles"). If the patient improves after 4-6 weeks, we recommend tapering prednisone, while continuing administration of antiviral agents for another 4-6 weeks. Long-term antiviral drugs are far less risky than long-term corticosteroids. Furthermore, if during a prednisone taper, patients' symptoms recur or worsen, along with increases in ESR or CRP, oral antivirals should be added rather than increasing the prednisone dose. In our experience, antiviral treatment has successfully normalized symptoms and inflammatory markers. In the future, rigorous prospective studies are still needed to determine whether oral antiviral agents and corticosteroids are as effective as intravenous acyclovir and corticosteroids, as well as appropriate dosage and duration of treatment.

In 2017, the US Food and Drug Administration approved the use of subcutaneous tocilizumab, an interleukin-6 (IL-6) receptor alpha inhibitor, for the treatment of GCA. The rationale for this treatment was that concentrations of CRP and other acutephase reactants that are increased by elevated serum levels of IL- 6 correlate with disease activity. Tocilizumab, combined with a 26-week prednisone taper, was superior to either 26-week or 52 -week prednisone tapering plus placebo with regard to sustained glucocorticoid-free remission in patients with GCA [24]. It is important to note that these current therapies for GCA target the inflammation that produces vascular damage, not the underlying cause of inflammation, which may account for relapses when therapies are discontinued. In virologically verified intracerebral VZV vasculopathy and GCA, VZV-infected vascular cells produce significantly elevated levels of IL-6, as well as other proinflammatory cytokines $[25,26]$; so while IL- 6 activity can be blocked and inflammation-mediated damage mollified by tocilizumab and corticosteroids, treatment of underlying VZV infection is essential, particularly because corticosteroids alone can potentiate VZV replication.

\section{GRANULOMATOUS ARTERITIS OFTHE AORTA AND TAKAYASU ARTERITIS}

Because granulomatous arteritis of the aorta has similar pathology to VZV vasculopathy and GCA, a smaller study similar to the GCA study by Gilden and colleagues [19] was conducted, which showed VZV antigen in 11 of 11 aortas with pathologically verified granulomatous arteritis, in 1 of 1 cases of nongranulomatous arteritis, and in 5 of 18 control aortas (28\%) obtained at autopsy, again most likely due to subclinical reactivation or early disease [27]. Studies with larger sample sizes for this rare disease still need to be completed to determine accurate percentages of aortas containing VZV antigen; however, clinicians 
should consider VZV vasculopathy in their differential diagnosis, particularly if associated with zoster.

A single case report raised the possibility of VZV infection in Takayasu arteritis [28]. The patient presented with GCA and Takayasu arteritis; VZV antigen was found in her TA biopsy. Acyclovir treatment led to dramatic clinical improvement with the patient able to leave hospice care. Thus, in these challenging cases, VZV vasculopathy should be on the differential diagnosis because antiviral treatment can potentially improve outcomes.

For both granulomatous arteritis of the aorta and Takayasu arteritis, testing for serum VZV DNA or anti-VZV antibodies (elevated anti-VZV IgM or 4-fold increase in anti-VZV IgG titers) should be considered but the utility and sensitivity for diagnosing VZV infection in these 2 diseases is unknown. Larger studies on the role of VZV infection and antiviral therapy need to be conducted but is quite challenging due to the rare nature of these diseases.

\section{PATHOGENESIS}

Because no animal model exists for stroke caused by VZV vasculopathy, pathogenesis studies have been restricted to arteries from subjects with VZV vasculopathy and primary human cerebrovascular cells infected with VZV. Immunohistochemical analysis of cerebral and TAs from 3 patients with virologically confirmed VZV vasculopathy revealed VZV antigen in the outermost adventitial layer of the artery early in infection and in the media and intima later in the course of disease, consistent with transaxonal spread of reactivated VZV to the arterial adventitia followed by transmural spread of virus [29]. VZV-infected arteries contained: (1) a disrupted internal elastic lamina; (2) a thickened intima composed of myofibroblasts expressing alpha-smooth muscle actin, potentially contributing to luminal narrowing/occlusion and ischemic stroke; and (3) a paucity of medial smooth muscle cells leading to loss of vessel wall integrity [29]. The expression of myosin in some of the myofibroblasts indicate that these cells may have originated from the media. VZV-infected arteries contained $\mathrm{CD} 4^{+}$and $\mathrm{CD} 8^{+} \mathrm{T}$ cells, $\mathrm{CD} 68^{+}$ macrophages and rare $\mathrm{B}$ cells expressing $\mathrm{CD} 20$ distributed in the adventitia and intima, but not the media [30]. Arteries from early VZV vasculopathy contained abundant neutrophils in the adventitia, which were absent in late VZV vasculopathy. Inflammatory cells were absent in control arteries.

The presence of neutrophils in the arterial adventitia in early VZV vasculopathy is consistent with their presence in the CSF of patients with neurological disease due to VZV [31-33]. Although the mechanisms of vascular remodeling triggered by VZV are unknown, neutrophils may play a role because they produce reactive oxygen species in response to infection, which may mediate smooth muscle cell proliferation and migration $[34,35]$ and induce apoptosis and loss of vascular smooth muscle cells $[36,37]$. Neutrophils also secrete elastase and matrix metalloproteinases (MMPs). In conjunction with activated MMPs directly secreted by VZV-infected vascular cells [38], these enzymes can lead to extracellular matrix breakdown, weakening of the vessel wall, and aneurysm formation [39-41]. Finally, a thickened intima was associated with inflammation in vasa vasorum vessels in early VZV vasculopathy, consistent with the notion that inflammatory cells secrete soluble factors that contribute to pathological vascular wall remodeling $[42,43]$.

In an attempt to identify biomarkers for VZV vasculopathy, CSF from 30 patients with virologically confirmed VZV vasculopathy was analyzed for levels of proinflammatory cytokines and MMPs [25]. As positive controls for CNS inflammatory disease, CSF from 30 patients with multiple sclerosis (MS) was studied, along with CSF from 20 healthy individuals to serve as negative controls. Compared to CSF of both MS and healthy controls, CSF from VZV vasculopathy patients had significant elevations in IL-8, IL-6, and MMP-2. These results might help explain the abundance of neutrophils and macrophages observed in VZV vasculopathy patients, because IL-8 is a chemoattractant for neutrophils and IL- 6 promotes macrophage differentiation. Increased levels of IL- 8 and IL- 6 along with MMP- 2 could contribute to the inflammation and vascular wall damage that are hallmarks of VZV vasculopathy.

A recent study to identify mechanisms contributing to the persistence of inflammatory cells in VZV-infected arteries months after disease onset focused on the dysregulated expression of programmed death ligand-1 (PD-L1) during VZV infection of primary vascular cells in vitro [44]. PD-L1 is a $40-\mathrm{kDa}$ type 1 transmembrane protein in the $\mathrm{B} 7$ immunoglobulin family that can be expressed on virtually all nucleated cells. It acts to suppress the immune system through interaction with its receptor, programmed cell death protein 1 (PD-1), which is expressed specifically on activated $T$ cells, B cells, and macrophages. VZV infection of various vascular cells in vitro lead to downregulation of PD-L1 expression, which only occurred after the VZV-mediated downregulation of major histocompatibility complex-I (MHC-I). Downregulation of PD-L1 could promote persistent inflammation in the infected arteries and explain how immune cells persist up to 10 months after diagnosis, while the initial downregulation of MHC-I could prevent viral clearance. These studies also identified a VZV-mediated downregulation of MHC-I in uninfected bystander cells that would further inhibit viral clearance in the infected arteries. Future studies on patient arteries infected with VZV are needed to confirm these in vitro findings.

\section{CONCLUSIONS}

VZV vasculopathy is a potentially treatable cause of vascular disease and should be suspected in patients with zoster or varicella followed by transient ischemic attacks, ischemic and hemorrhagic stroke, aneurysm, sinus thrombosis, and GCA. It is important to recognize that the absence of rash, a CSF pleocytosis, or VZV DNA in CSF does not exclude the diagnosis, particularly in immunosuppressed individuals. Characteristic features include imaging and angiographic abnormalities involving both 
large and small vessels. The best test for diagnosis is detection of anti-VZV IgG in the CSF. Treatment consists of intravenous acyclovir, as discussed in the VZV vasculopathy and GCA sections. While the mechanisms of VZV vasculopathy have not been fully elucidated, histological and immunohistochemical studies of VZV-infected arteries, analysis of CSF from VZV vasculopathy patients for cytokines and MMPs, and analysis of VZVinfected primary human vascular cells for MMPs, PD-L1, and MHC-1 provide clues to the pathogenesis of disease. Specifically, upon VZV reactivation from sensory and/or autonomic ganglia, virus travels along nerve fibers that terminate in the outermost adventitial layer of the artery where virus-infected adventitial fibroblasts elicit a robust inflammatory response involving neutrophils early in disease, and T cells and macrophages throughout the entire course of disease. VZV-infected cells, neutrophils, and other infiltrating immune cells produce activated MMPs, which degrade the extracellular matrix potentially leading to weakening of the vessel wall, aneurysm formation, and rupture. Soluble factors secreted by inflammatory cells further contribute to vascular smooth muscle death and accumulation of myofibroblasts in the thickened intima, potentially leading to occlusion of blood flow and ischemic stroke. Finally, downregulation of PD-L1 in $\mathrm{VZV}$-infected vascular cells may contribute to the persistence of inflammatory cells and downregulation of MHC-1 may prevent effective viral antigen presentation to immune cells.

\section{Notes}

Acknowledgments. The authors thank Marina Hoffman for editorial review and Cathy Allen for manuscript preparation.

Financial support. This work was supported by the National Institutes of Health (grant numbers NS094758 and AG032958 to M. A. N.).

Supplement sponsorship. This work is part of a supplement sponsored by the Royal Society of Medicine (Royal Charter number RC000525) funded through unrestricted educational grants from Merck, Sanofi Pasteur MSD, The Research Foundation for Microbial Diseases of Osaka University, Seqirus and GlaxoSmithKline.

Potential conflicts of interest. Both authors: No reported conflicts of interest. Both authors have submitted the ICMJE Form for Disclosure of Potential Conflicts of Interest. Conflicts that the editors consider relevant to the content of the manuscript have been disclosed.

\section{References}

1. Richter ER, Dias JK, Gilbert JE 2nd, Atherton SS. Distribution of herpes simplex virus type 1 and varicella zoster virus in ganglia of the human head and neck. J Infect Dis 2009; 200:1901-6.

2. Baudouin E, Lantuejoul P. Les troublecas moteurs dans le zona. Gazette des Hopitaux 1919.

3. Gilden DH, Kleinschmidt-DeMasters BK, Wellish M, Hedley-Whyte ET, Rentier B, Mahalingam R. Varicella zoster virus, a cause of waxing and waning vasculitis: the New England Journal of Medicine case 5-1995 revisited. Neurology 1996; 47:1441-6.

4. Lian Z, Zhu Y, Tang F, Yang B, Duan R. Herpes zoster and the risk of ischemic and hemorrhagic stroke: a systematic review and meta-analysis. PLosOne 2017; 12:e0171182.

5. Langan SM, Minassian C, Smeeth L, Thomas SL. Risk of stroke following herpes zoster: a self-controlled case-series study. Clin Infect Dis 2014; 58:1497-503.

6. Minassian C, Thomas SL, Smeeth L, Douglas I, Brauer R, Langan SM. Acute cardiovascular events after herpes zoster: a self-controlled case series analysis in vaccinated and unvaccinated older residents of the United States. PLoS Med 2015; 12:e1001919.
7. Nagel MA, Cohrs RJ, Mahalingam R, et al. The varicella zoster virus vasculopathies: clinical, CSF, imaging, and virologic features. Neurology 2008; 70:853-60.

8. Ciccone S, Faggioli R, Calzolari F, Sartori S, Calderone M, Borgna-Pignatti C. Stroke after varicella-zoster infection: report of a case and review of the literature. Pediatr Infect Dis J 2010; 29:864-7.

9. Sabry A, Hauk PJ, Jing H, et al. Vaccine strain varicella-zoster virus-induced central nervous system vasculopathy as the presenting feature of DOCK8 deficiency. J Allergy Clin Immunol 2014; 133:1225-7.

10. Liberman AL, Nagel MA, Hurley MC, Caprio FZ, Bernstein RA, Gilden D. Rapid development of 9 cerebral aneurysms in varicella-zoster virus vasculopathy. Neurology 2014; 82:2139-41.

11. Siddiqi SA, Nishat S, Kanwar D, Ali F, Azeemuddin M, Wasay M. Cerebral venous sinus thrombosis: association with primary varicella zoster virus infection. J Stroke Cerebrovasc Dis 2012; 21:917.e1-4.

12. Wilck MB, Zuckerman RA; AST Infectious Diseases Community of Practice. Herpes simplex virus in solid organ transplantation. Am J Transplant 2013; 13(Suppl 4):121-7.

13. Ma-Krupa W, Jeon MS, Spoerl S, Tedder TF, Goronzy JJ, Weyand CM. Activation of arterial wall dendritic cells and breakdown of self-tolerance in giant cell arteritis. J Exp Med 2004; 199:173-83.

14. Al-Abdulla NA, Rismondo V, Minkowski JS, Miller NR. Herpes zoster vasculitis presenting as giant cell arteritis with bilateral internuclear ophthalmoplegia. Am J Ophthalmol 2002; 134:912-4.

15. Al-Abdulla NA, Kelley JS, Green WR, Miller NR. Herpes zoster vasculitis presenting as giant cell arteritis with choroidal infarction. Retina 2003; 23:567-9.

16. Nagel MA, Khmeleva N, Boyer PJ, Choe A, Bert R, Gilden D. Varicella zoster virus in the temporal artery of a patient with giant cell arteritis. J Neurol Sci 2013; 335:228-30.

17. Victor DI, Green WR. Temporal artery biopsy in herpes zoster ophthalmicus with delayed arteritis. Am J Ophthalmol 1976; 82:628-30.

18. Nagel MA, Bennett JL, Khmeleva N, et al. Multifocal VZV vasculopathy with temporal artery infection mimics giant cell arteritis. Neurology 2013; 80:2017-21.

19. Gilden D, White T, Khmeleva N, et al. Prevalence and distribution of VZV in temporal arteries of patients with giant cell arteritis. Neurology 2015; 84:1948-55.

20. Gilden D, White T, Khmeleva N, Boyer PJ, Nagel MA. VZV in biopsy-positive and -negative giant cell arteritis: analysis of 100+ temporal arteries. Neurol Neuroimmunol Neuroinflamm 2016; 3:e216.

21. Muratore F, Croci S, Tamagnini I, et al. No detection of varicella-zoster virus in temporal arteries of patients with giant cell arteritis. Semin Arthritis Rheum 2017; 47:235-40.

22. Procop GW, Eng C, Clifford A, et al. Varicella zoster virus and large vessel vasculitis, the absence of an association. Pathog Immun 2017; 2:228-38.

23. Buckingham EM, Foley MA, Grose C, et al. Identification of herpes zoster-associated temporal arteritis among cases of giant cell arteritis. Am J Ophthalmol 2018; 187:51-60.

24. Stone JH, Tuckwell K, Dimonaco S, et al. Trial of tocilizumab in giant-cell arteritis. N Engl J Med 2017; 377:317-28.

25. Jones D, Alvarez E, Selva S, Gilden D, Nagel MA. Proinflammatory cytokines and matrix metalloproteinases in CSF of patients with VZV vasculopathy. Neurol Neuroimmunol Neuroinflamm 2016; 3:e246.

26. Jones D, Neff CP, Palmer BE, Stenmark K, Nagel MA. Varicella zoster virus-infected cerebrovascular cells produce a proinflammatory environment. Neurol Neuroimmunol Neuroinflamm 2017; 4:e382.

27. Gilden D, White T, Boyer PJ, et al. Varicella zoster virus infection in granulomatous arteritis of the aorta. J Infect Dis 2016; 213:1866-71.

28. Gilden D, White TM, Nagae L, Gurdin WH, Boyer PJ, Nagel MA. Successful antiviral treatment of giant cell arteritis and Takayasu arteritis. JAMA Neurol 2015; 72:943-6.

29. Nagel MA, Traktinskiy I, Azarkh Y, et al. Varicella zoster virus vasculopathy: analysis of virus-infected arteries. Neurology 2011; 77:364-70.

30. Nagel MA, Traktinskiy I, Stenmark KR, Frid MG, Choe A, Gilden D. Varicellazoster virus vasculopathy: immune characteristics of virus-infected arteries. Neurology 2013; 80:62-8.

31. Devinsky O, Cho ES, Petito CK, Price RW. Herpes zoster myelitis. Brain 1991; 114:1181-96.

32. Haug A, Mahalingam R, Cohrs RJ, Schmid DS, Corboy JR, Gilden D. Recurrent polymorphonuclear pleocytosis with increased red blood cells caused by varicella zoster virus infection of the central nervous system: case report and review of the literature. J Neurol Sci 2010; 292:85-8.

33. Stevens DA, Ferrington RA, Jordan GW, Merigan TC. Cellular events in zoster vesicles: relation to clinical course and immune parameters. J Infect Dis 1975; 131:509-15.

34. Hartney T, Birari R, Venkataraman S, et al. Xanthine oxidase-derived ROS upregulate Egr-a via ERK1/2 in PA smooth muscle cells; model to test impact of extracellular ROS in chronic hypoxia. PLoS One 2011; 6:e27531. 
35. Weber DS, Taniyama Y, Rocic P, et al. Phosphoinositide-dependent kinase 1 and p21-activated protein kinase mediate reactive oxygen species-dependent regulation of platelet-derived growth factor-induced smooth muscle cell migration. Circ Res 2004; 94:1219-26.

36. Hsieh CC, Yen MH, Yen CH, Lau YT. Oxidized low density lipoprotein induces apoptosis via generation of reactive oxygen species in vascular smooth muscle cells. Cardiovasc Res 2001; 49:135-45.

37. Li J, Li W, Su J, Liu W, Altura BT, Altura BM. Hydrogen peroxide induces apoptosis in cerebral vascular smooth muscle cells: possible relation to neurodegenerative diseases and strokes. Brain Res Bull 2003; 62:101-6.

38. Nagel MA, Choe A, Rempel A, Wyborny A, Stenmark K, Gilden D. Differential regulation of matrix metalloproteinases in varicella zoster virus-infected human brain vascular adventitial fibroblasts. J Neurol Sci 2015; 358:444-6.

39. Ferry G, Lonchampt M, Pennel L, de Nanteuil G, Canet E, Tucker GC. Activation of MMP-9 by neutrophil elastase in an in vivo model of acute lung injury. FEBS Lett 1997; 402:111-5.
40. Itoh Y, Nagase H. Preferential inactivation of tissue inhibitor of metalloproteinases- 1 that is bound to the precursor of matrix metalloproteinase 9 (progelatinase B) by human neutrophil elastase. J Biol Chem 1995; 270:16518-21.

41. Okada Y, Nakanishi I. Activation of matrix metalloproteinase 3 (stromelysin) and matrix metalloproteinase 2 ('gelatinase') by human neutrophil elastase and cathepsin G. FEBS Lett 1989; 249:353-6.

42. Frid MG, Brunetti JA, Burke DL, et al. Hypoxia-induced pulmonary vascular remodeling requires recruitment of circulating mesenchymal precursors of a monocyte/macrophage lineage. Am J Pathol 2006; 168:659-69.

43. Stenmark KR, Frid MG, Yeager M, et al. Targeting the adventitial microenvironment in pulmonary hypertension: a potential approach to therapy that considers epigenetic change. Pulm Circ 2012; 2:3-14.

44. Jones D, Blackmon A, Neff CP, et al. Varicella-zoster virus downregulates programmed death ligand 1 and major histocompatibility complex class I in human brain vascular adventitial fibroblasts, perineurial cells, and lung fibroblasts. J Virol 2016; 90:10527-34 